\title{
National Provider Identifier
}

National Cancer Institute

\section{Source}

National Cancer Institute. National Provider Identifier. NCI Thesaurus. Code C54633.

A unique identification number for health care services, supplies, and equipment providers in the United States. NPI is assigned for use in all administrative and financial transactions specified by HIPAA. NPI is issued by the National Plan and Provider Enumeration System (NPPES) and is 10-position, all-numeric identification number with no embedded intelligence. 